\title{
Node Synchronization Algorithm in Wireless Sensor Networks
}

\author{
Sagar S. Joshi \\ Department of Electronics \&Telecommunication, \\ Sinhgad institute of technology, Lonavala
}

\author{
D.S. Mantri \\ Department of Electronics \&Telecommunication, \\ Sinhgad institute of technology, Lonavala
}

\begin{abstract}
Inside a wireless sensor network there exist a number of nodes; often deployed in remote and inaccessible areas. These nodes take the power from batteries. The nodes are intended for monitoring physical phenomena like temperature changes, Percentage of humidity etc. Over the time, any of the nodes finds itself in a situation that it is difficult to maintain its course of routine. This can happen due to a discharged battery or any physical damage to the node. This situation hampers the synchronization accuracy among nodes. Therefore, in this paper a method is suggested to keep the throughput inside a wireless sensor network, simply by anticipating the situation with an algorithm that is based on consensus approach and using average time synchronization protocol.
\end{abstract}

\section{General Terms}

Wireless sensor network, synchronization

\section{Keywords}

Throughput, consensus, average time synchronization

\section{INTRODUCTION}

Today wireless sensor nodes are readily available at comparatively lower costs and with less power requirements. The areas where a proper wired communication infrastructure cannot be deployed e.g. islands; wireless sensor networks prove to be an assuring solution. Once deployed over the area they continuously monitor the physical parameters. These parameters include temperature, wind speed, tidal movement, humidity etc. By use of the inbuilt sensors, these nodes convert the physical quantities into discrete samples of signal. These discrete values or more precisely, the digital values are useful further for processing, forecast or simply storage [3]. The nodes; as mentioned first convert them into a meaningful way and then put these values on wireless radio links. These wireless communication links take this information to a proper means of collection. It is actually called a data sink or a gateway.

In order to correlate this information coming from nodes, all of them must be synchronized to a common time period.

This helps understand the data coming from each node and also at what interval of time, it arrives. A precisely synchronized network gives the guaranty of faithful operations. The nodes may be externally synchronized or internally synchronized. The nodes are externally synchronized means that they are guided to synchronize their own local clock to a common reference of time [4]. This common reference of time is an external reference clock. It must be an accurate real time standard. If they are internally synchronized then they have a proper idea of each other; with reference to their clocks. In this case, the aim is to obtain, consistent view of time across all the nodes in the network. If, the nodes get synchronized externally, at the same time they get internally synchronized with twice the precision as that of external synchronization [5].

The thing to be focused on is internal or local clock synchronization. The approach for synchronizing the nodes is chosen to be the consensus based [1]. Use of consensus approach fulfills the requirement that failure of single node does not affect the whole network operation. The protocol used is the average time synchronization protocol. This protocol is useful as it looks after not only the frequency but also the offset of the node clock [2].

The introductory part terminates here. Upcoming part of the paper illustrates the average time synchronization protocol, structure of node, algorithm, simulation and result followed by conclusion and references.

\section{AVERAGE TIME SYNCHRONIZATION}

When we apply the average time synchronization protocol, three main steps are followed. They include relative skew estimation, the skew compensation and offset compensation. In relative skew estimation, each node finds out the relative skew with respect to its neighbors. The process is carried out by first storing current local time into a broadcast packet. Second, the node receiving this packet records its own time at that particular instant. This same procedure happens when another package is received from the previous node.

In skew compensation, each node is forced to converge to a single virtual reference for a given wireless sensor network.

The local information now needs to be exchanged. It is given the preference. This is because of use of the distributed consensus algorithm [2]. More strong connected graphs allow finding a path between any nodes to any other node in the network. The actual value of reference time to which all the node clocks converge; depends upon the initial conditions and on communication topology.

In offset compensation, only the remaining offset errors are compensated. Here, use of consensus based approach is important as far as the need arises to update the virtual clock offset.

The consensus approach is used along with average time synchronization, based on the process of interchanging messages related to node synchronization. Therefore in case of any node occurring failure situation can be anticipated. Through this there arises the unknown time reference. This time reference is actually used by all the nodes while operating in normal situations.[1]

The average time synchronization algorithm uses following equation to find local time values of given node:

$$
\tau i(t)=[\alpha i t]+\beta i
$$


The equation gives the local hardware clock time of a node inside the network. In it, $\tau i(t)$ stands for local hardware clock time while $\alpha \mathrm{i}$ is the frequency and $\beta \mathrm{i}$ is the offset of the clock. While the equation for software parameters is given as:

$$
\hat{\tau} i(t)=\hat{\alpha} i(t)+\hat{o} i(t)
$$

\subsection{Structure of node}

When the term a wireless sensor node appears then it is important to visualize the structure of that sensor node. While deciding the proceedings of this paper the node was said to have following integral parts:

- First important part is the clock section. This section decides the duty cycle for node. That is, the time for any node to be on or off.

- Second part is the entity taking all the decisions; which in fact is a microprocessor. It takes all the decisions.

- Third important part is the radio section. This section is responsible for any communication in the neighborhood.

Here is a diagram showing the interconnections. In the structure, we can see that microprocessor decides all the operations going on and hence all the important decisions.

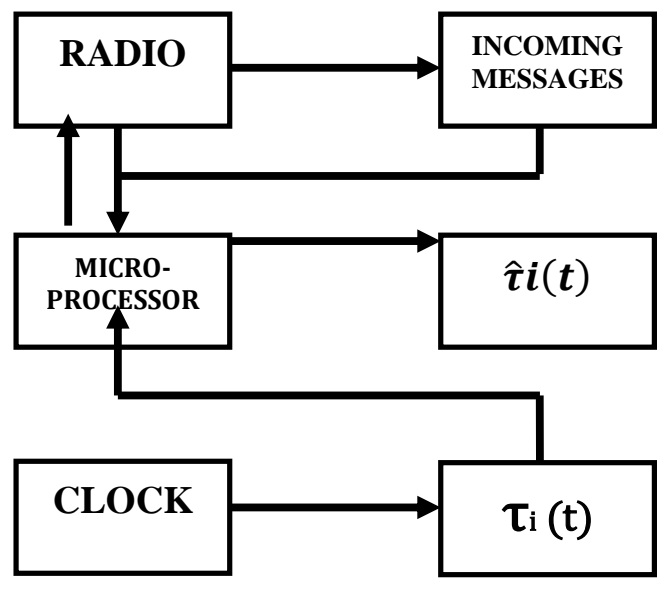

Figure 1

\section{ALGORITHM}

The flow of steps that any node is going to follow or the algorithm for the mentioned theoretical procedures is listed here after:

- Record the time interval from constituent nodes.

- Find the standard deviation of these clock periods/ time periods by using predefined formulae.

- Compare these values with standard/ practical values.

- If any node shows this value of standard deviation less than the standard value then this is acceptable value. If not then that node is harmful.

- Then consider the hardware and software clock times of this node.

- Isolate this node and it now needs to be fixed.
- Allow the node to refix its values. After getting the perfect values of synchronization parameters, the node can again be left inside the communication stream.

\section{SIMULATION AND RESULTS}

The simulations are carried out in order to decide whether the given algorithm works properly or not. Inside simulation environment, total 40 nodes will be deployed at random positions. The node 0 acts as sender and node 1 act as receiver. Each node sends a query request to its neighbor at the start of synchronization procedure. The routing protocol is ad-hoc on demand distance vector routing so that the route is not fixed and it will be decided depending upon weights assigned to it. The nodes are also categorized depending upon their share in overall procedure. The guard nodes are those nodes which monitor for the status of nodes under the path followed by data packets. These nodes forwarding data packets will be called as the routers.

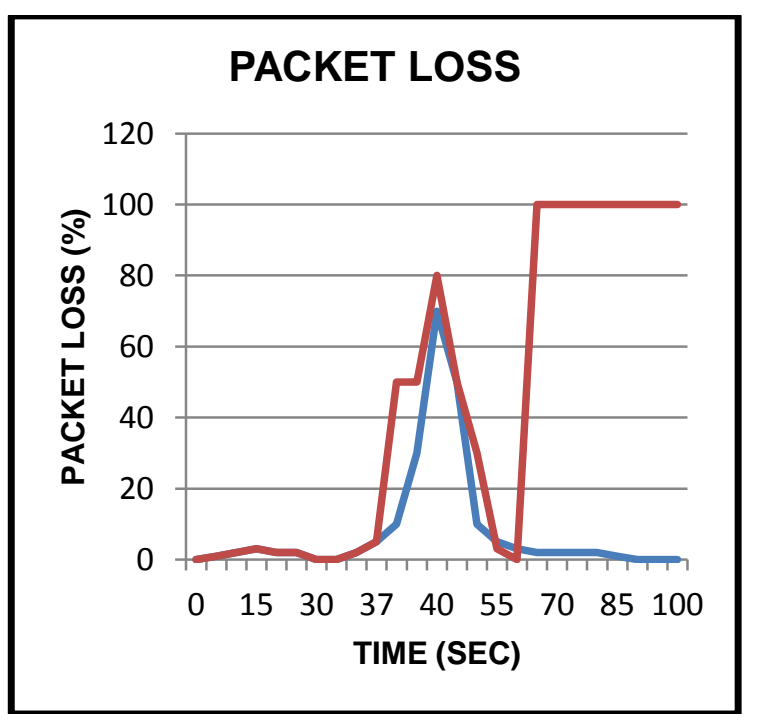

Figure 2

The function of guard nodes, as their name indicates is to monitor and safeguard the ongoing network operations. If any of the routers fails then guard node accuses it and decides to choose another optimal path to route the packets inside the network. Thus it keeps the throughput, packet loss and packet delivery ratio at required level.

The decision taken, whether a given node is faulty or not is decided by using the points mentioned in the algorithm earlier. If the node has a time values differing a lot from those of other nodes inside a network, that node is considered as faulty and is then isolated from all ongoing network operations.

While carrying out the simulations various parameters were selected and then corresponding results were found out. They are as following:

- Time taken by simulation from start to end: 100 seconds

- Synchronization time interval : 2 seconds

- Any random node among routers turns faulty. Time taken before activation of faulty node is: $360 \mathrm{~ms}$

- Time taken for deciding alternate optimal path: $120 \mathrm{~ms}$ 
- Inside network all the packets are sent in broadcast. Value of frequency for proper nodes is decided as $1 \mathrm{kHz}$ with zero offset.

- $\quad$ For faulty node the value of offset varies from 0 to 100 . Thus it can be detected and isolated.

- The packet loss when a node goes faulty is $100 \%$. While use of proposed method shows reduced packet loss.

- The packet delivery ratio when a node is found faulty takes the values as $0.00,0.05$ up to $0.15 \%$ over 100 seconds.

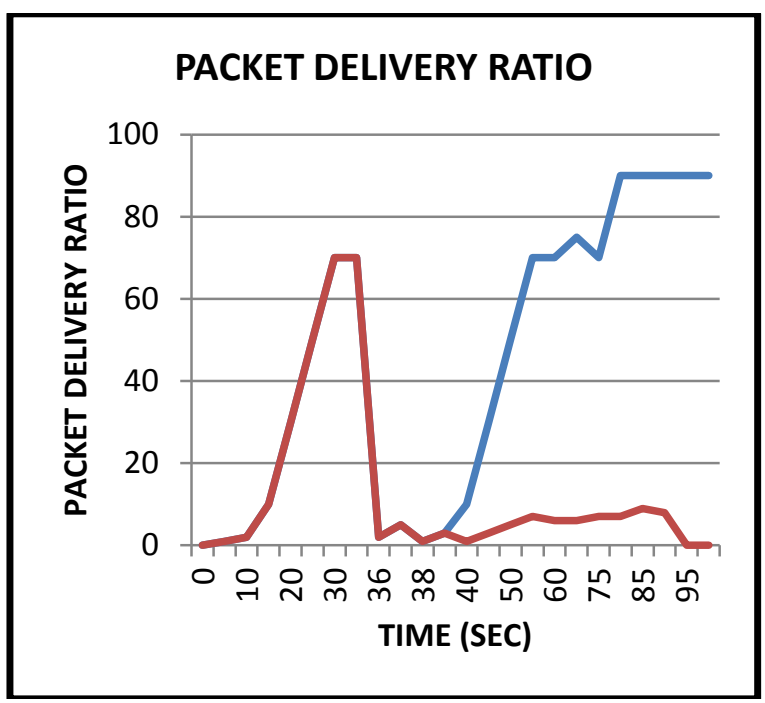

Figure 3

\section{ACKNOWLEDGEMENT}

It's a pleasure to thank Dr.M.S.GAIKWAD sir who has given the true direction for the entire work and his guidance is the key factor in developing the whole idea.

\section{CONCLUSION}

The paper focuses on a method to anticipate the issues arising when a faulty node comes across inside the network. Use of consensus approach along with average time synchronization proves healthy in such environment. It can not only detect the faulty node but also finds a way out for further operations. In future scope of paper, other consensus algorithms can be used with same idea for synchronizing nodes.

\section{REFERENCES}

[1] Francesco Lamonaca, Domenico Grimaldi," Synchronization for Hot-plugging Node in Wireless Sensor Network", 978-1-4673-2874-6/13/ 2013 IEEE.

[2] Luca Schenato, Alessio Basso," Average TimeSync (ATS): a distributed consensus protocol for sensor networks clock synchronization “.

[3] Achim Berger, Albert Pötsch, Andreas Springer, "Synchronized Industrial Wireless Sensor Network with IEEE 802.11 Ad Hoc Data Transmission."

[4] Na Wang, YanXia Pang ,"Time Synchronization for Wireless Sensor Network Based on Major Clock" , 978 1-4673-0242-5/12/\$31.00 @2012 IEEE.

[5] Wanseop Lee, Yongbin Yim, Soochang Park, Jeongcheol Lee, Hosung Park and Sang-Ha Kim, "A Cluster-based Continuous Object Tracking Scheme in Wireless Sensor Networks",978-1-4244-8327-3/11/\$26.00 @2011 IEEE. 